\title{
Dietary compensation in response to covert imposition of negative energy balance by removal of fat or carbohydrate
}

\author{
Gail R. Goldberg*, Peter R. Murgatroyd, Aideen P. M. McKenna, Patricia M. Heavey \\ and Andrew M. Prentice \\ MRC Dunn Clinical Nutrition Centre, Hills Road, Cambridge CB2 2DH, UK
}

(Received 25 September 1997 - Revised 12 January 1998 - Accepted 5 February 1998)

\begin{abstract}
Compensatory changes in energy intake (EI) and macronutrient metabolism in response to modest covert underfeeding were tested by whole-body calorimetry in eight lean men. Each was studied on three occasions comprising a controlled stabilization day followed by manipulation and outcome days in a whole-body calorimeter. On the manipulation day EI was fixed, and calculated to maintain energy balance (CONTROL) or to provide $85 \%$ of CONTROL by removing energy as carbohydrate (CHOred) or as fat (FATred). On the outcome day, ad libitum EI was allowed at fixed mealtimes. CHOred and FATred manipulations generated significantly different energy balances $(-1 \cdot 10$ (SE 0.13) MJ, $P=0 \cdot 000 ;-1 \cdot 10$ (SE 0.12) MJ, $P=0 \cdot 000)$ and fat balances ( $-0 \cdot 61$ (SE 0.23) MJ, $P=0 \cdot 03 ;-1.09$ (SE 0.20) MJ, $P=0 \cdot 000$ ), but not carbohydrate balances $(-0 \cdot 39$ (SE 0.22) MJ, NS; $0 \cdot 11$ (SE 0.23) MJ, NS) by the end of the manipulation day compared with CONTROL. On the outcome day, EI was significantly higher than CONTROL after CHOred (+1.58 (SE 0.33) MJ, $P=0.004)$ and FATred (+1.21 (SE 0.49) MJ, $P=0.022$ ) with no differences between treatments. Overall $48 \mathrm{~h}$ energy balances averaged close to zero at $-0 \cdot 14$, $+0 \cdot 34,+0 \cdot 04 \mathrm{MJ}$ on CONTROL, CHOred and FATred respectively. Total $48 \mathrm{~h}$ energy intakes on CHOred and FATred averaged 101 (SE 1.7) \% and 99 (SE 2.5) \% of CONTROL, thus demonstrating accurate detection of a mild energy deficit and efficient next-day compensation. Despite significant differences in macronutrient oxidation rates, the energy homeostatic mechanism appeared to be independent of specific macronutrient deficits.
\end{abstract}

Appetite: Homeostasis: Energy intake: Whole-body calorimetry

The regulation of energy and macronutrient balance is central to the control of body weight and adiposity but the mechanisms which regulate overall energy homeostasis remain to be established. Over the past decade it has become clear that obesity, and wasting due to disease, are more likely to be consequences of the failure to regulate appropriately energy intake rather than energy expenditure (Prentice et al. 1992). A number of hypotheses for the control of appetite have been proposed which are based on the regulation of energy (Friedman et al. 1990), fat (Kennedy, 1953; Hervey, 1969) carbohydrate (Russek, 1963; Flatt, 1987) and protein (Mellinkoff et al. 1956). Many different study designs have been used to test if and how humans are sensitive to covert dietary manipulations of energy and macronutrients (Poppitt \& Prentice, 1996).

In order to examine the glycoregulatory theories of Flatt (1987), we have previously used isoenergetic diets, in lean men at constant energy balance, to examine the relative importance of alterations in fat and carbohydrate intake or oxidation as mechanisms for rectifying extreme covert perturbations of glycogen stores (Stubbs et al. 1993; Shetty et al. 1994). An autoregulatory change in carbohydrate oxidation was the only significant mechanism for reestablishing carbohydrate balance; food intake was unaffected. In a further series of experiments we investigated the effects on food intake of large changes in the fat:carbohydrate ratio of covertly manipulated diets provided ad libitum to lean men over 7 to $14 \mathrm{~d}$ (Stubbs et al. 1995a,b, 1996). The key observation was that the subjects manifestly failed to detect the energy overload induced by the high-fat diet and went into marked positive fat balance $(+100 \mathrm{~g} / \mathrm{d})$. This occurred because they continued to eat the same physical bulk (weight) of food on each treatment irrespective of its energy density. One possible criticism of these and similar studies (Lissner et al. 1987; Kendall et al. 1991) is that physiologically-derived eating cues are so deeply embedded within cognitive, learned controls that they cannot be adequately tested using short-term experimental paradigms.

This suggestion is refuted by the study described here, in which we have tested the effects, again in lean men, of 
modest underfeeding by covertly removing dietary energy as fat or as carbohydrate, relative to a control treatment. The aims were to investigate the efficiency with which energy and macronutrient balances were restored in the $24 \mathrm{~h}$ period subsequent to the imposition of $15 \%$ negative energy balances, and to determine if different down-stream responses were elicited depending upon whether energy was removed as fat or carbohydrate.

\section{Methods}

Subjects

Nine healthy, non-obese male subjects, who lived and worked in the Cambridge area, participated in the study. Their occupations and leisure pursuits classified them as habitually sedentary or moderately active. Three subjects were light smokers. None had a history of fluctuating body weight. All subjects completed the study. However, we were concerned about one subject's ad libitum energy intake (EI) on the outcome day of the CONTROL run. The subject had participated in previous experiments and his ad libitum intake was atypically high. This subject's data have been excluded from the analysis as presented here. Inclusion of his data makes a small difference to some of the significance values but does not alter any of the interpretations. The mean characteristics of the eight remaining subjects were as follows: age 35.7 (SD 10.3) years; height 1.76 (SD 0.04) m; weight $70 \cdot 3(\mathrm{SD} 9 \cdot 5) \mathrm{kg}$; BMI $22 \cdot 8(\mathrm{SD} 2 \cdot 9) \mathrm{kg} / \mathrm{m}^{2}$. The subjects' weights did not alter over the course of the study.

\section{Study design}

Before the main study all subjects had their BMR measured under standard conditions (Goldberg et al. 1988). These data were used to calculate the dietary intakes. Subjects were then studied on three occasions each consisting of a maintenance day, manipulation day and outcome day. The maintenance day was spent in the Unit's metabolic suite to ensure that subjects were in the same metabolic state on each occasion. Only minimal physical activity was permitted to prevent significant changes in muscle glycogen stores, and EI was fixed at 1.35 $\times$ BMR. After their evening meal subjects entered a whole-body calorimeter where they followed a fixed activity protocol for the next $61 \mathrm{~h}$ (manipulation and outcome days and preceding night). On the manipulation day, subjects received diets calculated to maintain energy balance (CONTROL) or which were covertly manipulated to have a $15 \%$ energy deficit relative to CONTROL, achieved by removing energy in the form of fat (FATred) or carbohydrate (CHOred). CONTROL EI was fixed at $1.46 \times \mathrm{BMR}$, the level of energy expenditure anticipated on the activity protocol used. On the outcome days ad libitum intake of meals of the same macronutrient composition as CONTROL was allowed. The CONTROL treatment was completed first and the order of FATred and CHOred treatments was then randomized for each subject. The study was approved by the Dunn Nutrition Unit's Ethical Committee. Subjects gave their written consent to the methods used in the study, although due to the nature of the investigation they were not told its exact purpose until the end of the experiment.

\section{Diets}

Dietary intakes were calculated from food table values for metabolizable energy (Holland et al. 1991). EI was calculated to the nearest $0 \cdot 1 \mathrm{MJ}$ for each subject. Meals were composed of normal foods, weighed to the nearest $1 \mathrm{~g}$ and prepared in the Unit's metabolic kitchen. The maintenance, CONTROL and outcome diets provided $48 \%$, $40 \%$ and $12 \%$ energy from carbohydrate, fat and protein respectively. On CONTROL and FATred the absolute intakes (MJ) of carbohydrate were the same, whilst on CONTROL and CHOred the absolute fat intakes were the same. Protein intake remained constant across all treatments. On both the maintenance and manipulation days subjects were obliged to eat all the food provided. Each meal was identical in energy content and macronutrient composition and provided one-third of the total day's intake. The meals served on the manipulation day were very similar in appearance and bulk across treatments; manipulations were mainly achieved by small adjustments in the amounts of fruit, fruit juice, butter, margarine, cream and mayonnaise. Palatability was not a significant issue on the manipulation day since subjects were obliged to eat all foods.

On the outcome day, at mealtimes, subjects could eat as much as they liked. The method is one we have used in a number of previous calorimeter studies (Stubbs et al. 1993, 1995a; Shetty et al. 1994). Meals were all of the same macronutrient composition, were mostly homogeneous (breakfast cereal and milk, vegetable stew, fruit yoghurt, etc.) to prohibit macronutrient selection and were identical on all treatments to exclude palatability effects. Foods such as bread rolls and butter in which the components could not be mixed homogeneously had to be eaten as complete units. The foods at each meal were served in excess and the same amount was presented on all occasions to all subjects. The foods offered at lunch and the evening meal were identical. Thus subjects always ate meals of the same composition and in amounts of their choosing. The energy and macronutrients consumed were calculated from the difference between the weights of food served and leftover at each meal.

\section{Whole-body calorimetry and macronutrient oxidation}

A fixed protocol was followed which, in each $24 \mathrm{~h}$ period, included $8 \mathrm{~h}$ sleep, $1 \mathrm{~h}$ BMR, $2 \mathrm{~h}$ exercise $(2 \times 30 \mathrm{~min}$ stepping on and off a $220 \mathrm{~mm}$ block; $2 \times 30 \mathrm{~min}$ at a workload of $25 \mathrm{~W}$ on a cycle ergometer); and $3 \times 30$ min mealtimes. The remainder of the time was spent in sedentary activities. Subjects were closely supervised at all times by the investigators or a night nurse. The experiments were performed in three whole-body calorimeters previously described (Murgatroyd et al. 1993; Jebb et al. 1996). Rapid response calculations were used to calculate $\mathrm{O}_{2}$ consumption and $\mathrm{CO}_{2}$ production (Brown et al. 1984). Urine samples were analysed for $\mathrm{N}$ content (Kjeltec Auto Sampler System 1035/38, Tecator, Höganäs, Sweden or Leco FP428 Nitrogen Analyser, Stockport, UK). Macronutrient 
oxidation rates were calculated from non-protein gaseous exchange, using the coefficients of Livesey \& Elia (1988). The limits of systematic error, estimated from gas infusion tests, were $\pm 10 \mathrm{~g} / \mathrm{d}$ for fat oxidation and $\pm 20 \mathrm{~g} / \mathrm{d}$ for carbohydrate oxidation. The precision of urinary $\mathrm{N}$ analysis from repeated measurements of a standard urea solution was $0.13 \mathrm{~g}$ (Murgatroyd et al. 1993). 24h data were calculated between 09.00-09.00 hours. Macronutrient balances (intake minus oxidation) were calculated relative to a nominal zero at 09.00 hours (representing the overnight fasted state). All analyses were performed with the macronutrients expressed as energy. Energy densities of 16, 37 and $17 \mathrm{~kJ} / \mathrm{g}$ were assumed for intakes of carbohydrate, fat and protein. Total energy intakes, expenditures and balances were calculated from the sum of the macronutrient values.

\section{Statistics}

Statistical analyses were performed using DataDesk Statistical Package, version 4.1r (Odesta Corporation, Northbrook, IL, US). Scheffé's post-hoc tests were used to test for differences between treatments and days. Values are expressed as means and SE or SD where indicated.

\section{Results}

Table 1 summarizes mean (SE) $24 \mathrm{~h}$ intakes, expenditures and balances of total energy and energy from carbohydrate and fat. There were no significant differences in protein oxidation or balance between or within treatments.

\section{Manipulation days}

As set up by the experimental design, energy intakes on CHOred and FATred were $15 \%$ lower than CONTROL. Energy expenditures on CHOred $(-0.40$ (SE 0.13$) \mathrm{MJ}$, $P=0.026)$ and FATred (-0.43 (SE 0.13) MJ, $P=0 \cdot 018$ ) were also significantly lower than CONTROL $(-4 \%)$. This somewhat offset the intended manipulation of $15 \%$ but energy balances on CHOred and FATred were still significantly lower than CONTROL $(-1 \cdot 10$ (SE 0.12), -1.09 (SE 0.13) MJ respectively; $P=0 \cdot 000$ for both). There were no significant differences in energy intake, expenditure or balance between CHOred and FATred.

Fat oxidation was significantly higher on CHOred $(+0 \cdot 61$ (SE 0.24) MJ, $P=0.030$ ) and marginally lower on FATred compared with CONTROL ( $-0 \cdot 40$ (SE 0.21) MJ, $P=0 \cdot 180)$. It differed significantly between CHOred and FATred (1.01 (SE 0.16) MJ, $P=0 \cdot 000$ ). Carbohydrate oxidation was significantly lower on CHOred compared with CONTROL $(-1 \cdot 10$ (SE 0.26) MJ, $P=0 \cdot 000)$ and CHOred compared with FATred $(-0.99(\mathrm{SE} 0 \cdot 18) \mathrm{MJ}, P=0 \cdot 002)$. Due to these adjustments in oxidation, neither manipulation generated significantly different carbohydrate balances compared with CONTROL on the manipulation day (CHOred -0.39 (SE $0 \cdot 23)$ MJ, $P=0 \cdot 210$; FATred $+0 \cdot 11$ (SE 0.23) MJ, $P=0 \cdot 873)$. However, both manipulations generated significantly different fat balances (CHOred $-0 \cdot 61$ (SE 0.24) MJ, $P=0 \cdot 030 ;$ FATred -1.09 (SE 0.22) MJ, $P=0 \cdot 000$ ). The differences in carbohydrate balance $(0.50$ (SE 0.16) MJ, $P=0 \cdot 091)$ and in fat balance (0.48 (SE 0.14) MJ, $P=0 \cdot 094)$ between CHOred and FATred were not significant.

\section{Outcome days}

On the outcome days, mean EI was significantly higher after both CHOred $(+1.58$ (SE 0.33) MJ, $P=0.004)$ and FATred $(+1.21(\mathrm{SE} 0 \cdot 49) \mathrm{MJ}, P<0.022)$ treatments than after CONTROL. The difference in intake between CHOred and FATred was not significant (0.36 (SE 0.30) MJ, $P=0 \cdot 643$ ). There was no difference in energy expenditure across treatments. Energy balances on CHOred and FATred were significantly higher than CONTROL $(+1.63$ (SE 0.26) MJ, $P=0.003$; +1.32 (SE 0.50) MJ, $P=0.0113$ respectively).

Macronutrient intake reflected total energy intake on the outcome days, because all meals were of identical composition. Thus compared with CONTROL, fat and carbohydrate intakes were significantly higher on CHOred (0.62 (SE $0 \cdot 13$ ) MJ and 0.76 (SE 0.16) MJ respectively, both $P=0 \cdot 004$ ) and on FATred (0.48 (SE 0.19) MJ, 0.58 (SE 0.24) MJ respectively, $P=0 \cdot 022)$. There were no significant differences in carbohydrate oxidation on either CHOred $(0 \cdot 47$ (SE $0 \cdot 32) \mathrm{MJ}, P=0 \cdot 177)$ or FATred (0.09 (SE 0.20) MJ, $P=0 \cdot 932)$ on the outcome day compared with CONTROL. Similarly there were no significant differences in fat oxidation between CONTROL and either CHOred (0.39 (SE 0.28) MJ, $P=0 \cdot 242$ ) or FATred (0.00 (SE 0.19) MJ, $P=1 \cdot 000$ ). Although on the outcome day carbohydrate oxidation was lower $(0 \cdot 38(\mathrm{SE} 0 \cdot 17) \mathrm{MJ})$ and fat oxidation was higher $(0.39$ (SE 0.18) MJ) on CHOred compared with FATred, the differences were not significant $(P=0.305$ and 0.241 respectively).

Fig. 1 illustrates the changes in energy, carbohydrate and fat balance induced on the manipulation day, the compensatory changes on the outcome day, and the resultant imbalance on both the CHOred and FATred treatments. In all cases the changes were adjusted by subtracting away the respective changes that occurred on the CONTROL treatment. This procedure should adjust the data for any potential systematic bias in estimating substrate oxidation rates. The figure demonstrates that energy compensation is generally superior to macronutrient compensation, particularly with respect to carbohydrate where there is a counter-regulatory adjustment on the FATred treatment.

\section{Comparison between manipulation and outcome days and 48 h data}

The positive energy balances on the outcome days were similar in magnitude to the negative balances on the manipulation days, resulting in $48 \mathrm{~h}$ energy balances averaging $-0 \cdot 14,+0 \cdot 34,+0 \cdot 04 \mathrm{MJ}$ on CONTROL, CHOred and FATred respectively. The differences across treatments were not significant. Overall $48 \mathrm{~h}$ carbohydrate balances were all positive, averaging 0.67 MJ (CONTROL), $1.51 \mathrm{MJ}$ (CHOred) and 1.46 MJ (FATred) whereas all fat balances were negative averaging $-0.89 \mathrm{MJ}$ (CONTROL), $-1.28 \mathrm{MJ}$ (CHOred) and $-1.51 \mathrm{MJ}$ (FATred). 


\section{Discussion}

After decades of research, many investigators now agree that physiological modulations in energy expenditure probably play a relatively minor role in the regulation of human energy balance. The scope for such modulations is certainly an order of magnitude smaller than possible changes in food intake which has re-focused attention on research into appetite control mechanisms. A key question is whether regulation is driven by lipostatic, gluco- or glycogenostatic or energostatic systems and indeed whether any of these physiological effectors have any significant homeostatic role in the face of the powerful social and environmental influences affecting modern man.

Most experiments which investigate appetite regulation use relatively short protocols consisting of a pre-load and subsequent test-meal(s) (Hill et al. 1995; Rolls \& Hammer, 1995). However, such methods are very sensitive to the time interval chosen because of temporal changes in the 'satiety cascade' (Blundell \& Halford, 1994). Furthermore the measurement of early (next meal) compensation may not reflect the full down-stream response. When experiments are extended to measure evening or next day intake they usually rely on self-completed food diaries rather than on precise quantification. Studies of longer duration conducted in whole-body calorimeters therefore have many advantages. Changes in appetite and food intake can be precisely monitored and related to exact changes in macronutrient and energy balance. The chamber provides a good environment for testing underlying physiological determinants of food intake because of its reproducible conditions and minimal external eating cues. Although this environment is highly artificial, the treatment effects of covert dietary manipulations with respect to voluntary food intake are similar to those obtained in free-living subjects (Stubbs et al. $1995 a, b)$. A persistent theme arising from our own calorimeter experiments has been the failure of subjects to alter their food intake in response to major manipulations of macronutrient intake when these are performed isoenergetically. Covert manipulations of fat content have also had no impact on voluntary food intake measured as the weight of food eaten.

The intention of this study was to maximize the likelihood of observing differences in intake in response to physiological cues, without introducing confounding or overriding variables. Subjects could eat ad libitum from meals which were palatable, but not to the extent that they would have induced cafeteria-type overeating. Only quantitative food selection was allowed, so providing diets of fixed composition did not allow us to discriminate any potential differences in the satiating properties of the different macronutrients. Macronutrient selection might have been observed if subjects had been given the opportunity to choose from a wide range of foods. However, it is far more likely that in a calorimeter they would have spontaneously overeaten by a considerable amount (the cafeteria diet effect), as we (M Totton, GR Goldberg, PR Murgatroyd and AM Prentice, unpublished results) and others (Larson et al. 1995) have previously observed.

In the present study modest underfeeding led to negative energy balances of almost $1.5 \mathrm{MJ}$ and the removal of only 


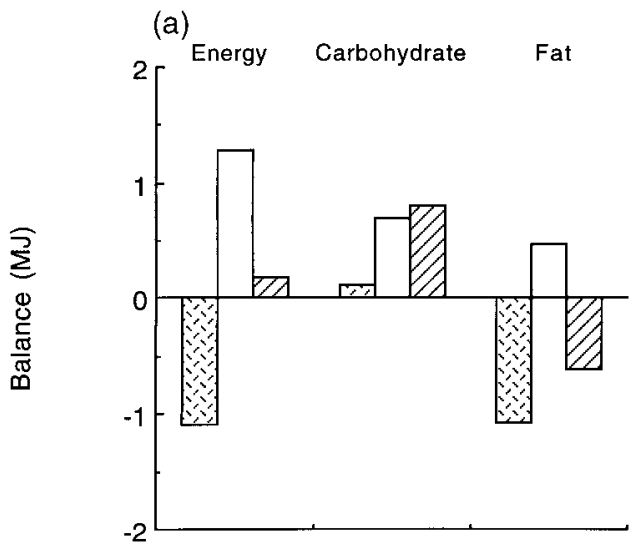

(b)

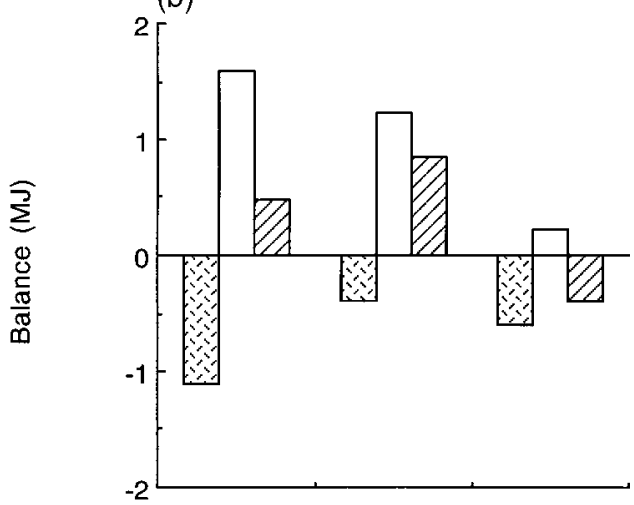

Fig. 1. Compensatory changes in energy, carbohydrate and fat balance following intentional mild energy restriction in young men. (a) Reduced-fat diet, (b) reduced-carbohydrate diet. (圆) Manipulation day; ( $\square$ ) outcome day; (四) resultant balance. All values calculated by subtracting respective changes on maintenance energy intake.

$15 \%$ of energy as carbohydrate or fat elicited significant increases in subsequent energy intake. The compensation was excellent; energy intakes on CHOred and FATred averaged $+15 \%$ and $+12 \%$ compared with CONTROL values. This actually represented a slight over-compensation because energy expenditure also decreased in response to the energy deficit. Thus the total compensation efficiencies on the outcome day were $125 \%$ for CHOred and $103 \%$ for FATred indicating an impressive average level of detection and correction for the energy deficit imposed on these lean men. Overall $48 \mathrm{~h}$ energy balances were close to zero (CHOred +0.34 MJ, FATred +0.04 MJ). There was no significant difference between the responses to carbohydrate or fat reduction which is in accord with a number of shorterterm studies in normal-weight young men showing that the effects of fat and carbohydrate on hunger, satiety or subsequent food intake are similar (Rolls \& Hammer, 1995). Compensation was mostly achieved by changes in food intake, but an unexpectedly large amount $(\sim 0.4 \mathrm{MJ})$ occurred through a decrease in energy expenditure, effectively reducing the intended manipulation to only $-11 \%$. This decrease is difficult to explain. The reduction in food intake of $1.5 \mathrm{MJ} / \mathrm{d}$ would only be expected to reduce diet-induced thermogenesis by about $0.15 \mathrm{MJ} / \mathrm{d}$; the calorimeter protocol is highly standardized (a fact reflected in the high level of significance for such a small change); the decrease cannot be attributed to differences in macronutrient composition because expenditure on CHOred and FATred treatments was identical. An order (training) effect is a possibility since the CONTROL treatment was always conducted first. However, this seems an unlikely explanation since expenditure on the outcome days reverted to the CONTROL levels. Thus the reduction in energy expenditure appears genuine and represents a partial explanation for the energy compensation.

The conclusion that accurate energy compensation was achieved through a physiological modulation of food intake is vulnerable to one possible confounding influence. The subjects might have recognized the deprivation and made a cognitive correction on the following day. We consider that to be unlikely because the meals were composed of similar food items and were similar in size. It is also unlikely that the form in which carbohydrate or fat was removed would have affected any habitual feelings of 'fullness', and therefore the amount of food eaten on the outcome day. In order to explain alterations in amounts of, for example, fruit juice, or the type of fat, subjects were told that the purpose of the study was to investigate the effects on energy expenditure of different kinds of fats and carbohydrate.

In our previous studies in which manipulations of carbohydrate were coincident with fat, we could not address the question of which was the more potent mediator of changes in fuel selection. Results from the present study suggest that the system is more sensitive to carbohydrate which is in accord with the studies of other authors in which carbohydrate intake was altered whilst fat intake was kept constant and vice versa (Flatt et al. 1985; Schutz et al. 1989; Griffiths et al. 1994). In line with our previous work and with predictions based on the macronutrient oxidative hierarchy (Prentice et al. 1992), the linkage between intake and oxidation was far closer for carbohydrate than for fat. On the manipulation day, when carbohydrate intakes were identical, there was no difference in carbohydrate oxidation between FATred and CONTROL. Removal of $1.5 \mathrm{MJ}$ carbohydrate led to a significant suppression of carbohydrate oxidation. Also, as in previous studies, the suppression of carbohydrate oxidation persisted into the outcome day despite reverting to an intake which had the same macronutrient composition as CONTROL. After the manipulation day carbohydrate balances were positive on all treatments, even CHOred. This is probably a short-term effect, since a negative balance may have resulted if the manipulation had been carried out for longer than $24 \mathrm{~h}$, or if a more extreme carbohydrate reduction had been imposed. The positive balances may also have been a result of the modest level of exercise in the calorimeter, immediately preceded by a very sedentary day which did not require any mobilization and oxidation of muscle glycogen.

In contrast to carbohydrate and consonant with its position at the bottom of the oxidative hierarchy, equivalent changes in fat intake had little impact on fat oxidation. On CHOred fat oxidation was significantly higher than on both FATred and CONTROL treatments because fat was substituting for the missing carbohydrate. The differences in fat oxidation led to significant negative fat balances on CHOred 
and FATred; further evidence that changes in fat oxidation and balances are driven by autoregulatory changes in carbohydrate intake and oxidation. There was no difference in fat oxidation on the outcome days indicating that correction of the negative fat balance represented a low priority compared with the correction of carbohydrate balance (see balance columns for outcome day in Table 1).

The disparate values for macronutrient compensation (including some counter-regulatory adjustments for carbohydrate) in this study lend strong general support to the concept of the energostatic model of regulation. Since both underfeeding treatments also generated significant negative fat balances which constituted most of the negative energy balance, the results also raise the possibility that the compensatory changes in intake could have been driven by lipostatic mechanisms.

We conclude from the results of the present study that in lean men, by definition subjects presumed to possess good regulatory mechanisms, appetite responds to small shortterm negative perturbations in fat balance. It could be argued that the ability to up-regulate energy intake efficiently in response to deficits will have been subjected to stronger evolutionary selection than the ability to downregulate since the former is essential to survival. To test this hypothesis we are currently repeating the experimental design used here with a $15 \%$ covert energy increment in the form of fat or carbohydrate.

\section{Acknowledgements}

We thank the volunteers for taking part in this study, Elaine Collard and Judith Wills for preparing the diets, and nightnurses Cathy Baker, Maxine Durham, Pauline Hordern and Heather Tulett.

\section{References}

Blundell JE \& Halford JC (1994) Regulation of nutrient supply: the brain and appetite control. Proceedings of the Nutrition Society 53, 407-418.

Brown D, Cole TJ, Dauncey MJ, Marrs RW \& Murgatroyd PR (1984) Analysis of gaseous exchange in open-circuit indirect calorimetry. Medical and Biological Engineering and Computing 22, 333-338.

Flatt JP (1987) The difference in the storage capacities for carbohydrate and for fat, and its implications in the regulation of body weight. Annals of the New York Academy of Sciences 499, 104-123.

Flatt JP, Ravussin E, Acheson KJ \& Jequier E (1985) Effects of dietary fat on postprandial substrate oxidation and on carbohydrate and fat balances. Journal of Clinical Investigation 76, 1019-1024.

Friedman MI, Ramirez I, Bowden CR \& Tordoff MG (1990) Fuel partitioning and food intake: role for mitochondrial fatty acid transport. American Journal of Physiology 258, R216-R221.

Goldberg GR, Prentice AM, Davies HL \& Murgatroyd PR (1988) Overnight and basal metabolic rates in men and women. European Journal of Clinical Nutrition 42, 137-144.

Griffiths AJ, Humphreys SM, Clark ML, Fielding BA \& Frayn KN (1994) Immediate metabolic availability of dietary fat in combination with carbohydrate. American Journal of Clinical Nutrition 59, 53-59.

Hervey GR (1969) Regulation of energy balance. Nature 222, $347-361$.
Hill AJ, Rogers PJ \& Blundell JE (1995) Techniques for the experimental measurement of human eating behaviour and food intake: a practical guide. International Journal of Obesity 19, 361-375.

Holland B, Welch AA, Unwin ID, Buss DH, Paul AA \& Southgate DAT (1991) McCance \& Widdowson's The Composition of Foods, 5th ed. Cambridge: Royal Society of Chemistry and Ministry of Agriculture, Fisheries and Food.

Jebb SA, Prentice AM, Goldberg GR, Murgatroyd PR, Black AE \& Coward WA (1996) Changes in macronutrient balance during overfeeding and underfeeding assessed by 12 -day continuous whole-body calorimetry. American Journal of Clinical Nutrition 64, 259-266.

Kendall A, Levitsky DA, Strupp BJ \& Lissner L (1991) Weight loss on a low-fat diet: consequence of the imprecision of the control of food intake in humans. American Journal of Clinical Nutrition 53, 1124-1129.

Kennedy GC (1953) The role of depot fat in the hypothalamic control of food intake in the rat. Proceedings of the Royal Society (B) 140, 578-592.

Larson DE, Rising R, Ferraro RT \& Ravussin E (1995) Spontaneous overfeeding with a 'cafeteria diet' in men: effects on 24hour energy expenditure and substrate oxidation. International Journal of Obesity 19, 331-337.

Lissner L, Levitsky DA, Strupp BJ, Kalkwarf HJ \& Roe DA (1987) Dietary fat and the regulation of energy intake in human subjects. American Journal of Clinical Nutrition 46, 886-892.

Livesey G \& Elia M (1988) Estimation of energy expenditure, net carbohydrate utilisation, and net fat oxidation and synthesis by indirect calorimetry: evaluation of errors with special reference to the detailed composition of fuels. American Journal of Clinical Nutrition 47, 608-628.

Mellinkoff S, Frankin M, Boyle D \& Geipell M (1956) Relationship between serum amino acid concentration and fluctuation in appetite. Journal of Applied Physiology 8, 535538.

Murgatroyd PR, Sonko BJ, Wittekind A, Goldberg GR, Ceesay SM \& Prentice AM (1993) Non-invasive techniques for assessing carbohydrate flux: 1. Measurement of depletion by indirect calorimetry. Acta Physiologica Scandinavica 147, 91-98.

Poppitt SD \& Prentice AM (1996) Energy density and its role in the control of food intake: Evidence from metabolic and community studies. Appetite 26, 153-174.

Prentice AM, Stubbs RJ, Sonko BJ, Diaz E, Goldberg GR, Murgatroyd PR \& Black AE (1992) Overview: Energy requirements and energy storage. In Energy Metabolism: Tissue Determinants and Cellular Corollaries, pp. 211-227 [J Kinney and $\mathrm{H}$ N Tucker, eds]. New York: Raven Press Ltd.

Rolls BJ \& Hammer VA (1995) Fat, carbohydrate, and the regulation of energy intake. American Journal of Clinical Nutrition 62 (Suppl.), 1086S-1095S.

Russek M (1963) An hypothesis on the participation of hepatic glucoreceptors in the control of food intake. Nature 197, 79-80.

Schutz Y, Flatt JP \& Jequier E (1989) Failure of dietary fat intake to promote fat oxidation: a factor favouring the development of obesity. American Journal of Clinical Nutrition 50, 307-314.

Shetty PS, Prentice AM, Goldberg GR, Murgatroyd PR, McKenna APM, Stubbs RJ \& Volschenk PA (1994) Alterations in fuel selection and voluntary food intake in response to isoenergetic manipulation of glycogen stores in man. American Journal of Clinical Nutrition 60, 534-543.

Stubbs RJ, Harbron CG, Murgatroyd PR \& Prentice AM (1995a) Covert manipulation of dietary fat and energy density: effect on substrate flux and food intake in men eating ad libitum. American Journal of Clinical Nutrition 62, 316-329.

Stubbs RJ, Harbron CG \& Prentice AM (1996) Covert manipulation of the dietary fat to carbohydrate ratio of 
isoenergetically dense diets: effect on food intake in feeding men ad libitum. International Journal of Obesity 20, 651-660.

Stubbs RJ, Murgatroyd PR, Goldberg GR \& Prentice AM (1993)

Carbohydrate balance and the regulation of day-to-day food intake in humans. American Journal of Clinical Nutrition 57, 897-903.
Stubbs RJ, Ritz P, Coward WA \& Prentice AM (1995b) Covert manipulation of the ratio of dietary fat to carbohydrate and energy density: effect on food intake and energy balance in free-living men eating ad libitum. American Journal of Clinical Nutrition 62, 330-337. 